AperTO - Archivio Istituzionale Open Access dell'Università di Torino

\title{
IMAGINE THERE'S NO (PLATONIC) HEAVEN
}

\section{This is a pre print version of the following article:}

Original Citation:

Availability:

This version is available http://hdl.handle.net/2318/1730691

since 2020-05-07T19:07:37Z

Published version:

DOI:10.1017/S1477175614000244

Terms of use:

Open Access

Anyone can freely access the full text of works made available as "Open Access". Works made available under a Creative Commons license can be used according to the terms and conditions of said license. Use of all other works requires consent of the right holder (author or publisher) if not exempted from copyright protection by the applicable law. 


\section{Penultimate draft, forthcoming in Think, published by Cambridge University Press, (C) Cambridge University Press.}

\section{Imagine there's no (platonic) heaven.}

Some people think that numbers and other mathematical entities exist. They believe in a platonic heaven of ideal mathematical objects, as some (other) people like to put it. This may seem a very strange thing to believe in: after all, we cannot see numbers, nor touch them, nor smell them. So why should one believe that they exist? Because, as Putnam and Quine used to say, numbers are indispensable to science: it seems almost impossible to state our best scientific theories without mentioning numbers or other mathematical objects.

Now suppose for a moment that believers in numbers (also called "platonists") are right on the latter point: we can't represent reality in a precise way without using mathematical vocabulary. Does this mean that we are compelled to think that numbers exist? It could be tempting to think that this is the case. After all, if mathematics is really indispensable to our description of the world, this means that you cannot describe a world as complex as the actual world without mentioning numbers: doesn't this mean that you could not even describe a world as complex as the actual world if you thought that numbers were absent in that world?

Stephen Yablo ${ }^{1}$ has recently gave us reason to doubt that this line of argument is correct. The argument for the existence of numbers we are considering has this form:

P. We can't represent a complex world without numbers.

therefore:

C. We can't represent a complex world lacking numbers.

Yablo rightly notes that this argument looks similar to a famous argument attributed to Berkeley:

P. we can't imagine a tree non-perceptually.

therefore:

C. we can't imagine unperceived trees.

Many people find this argument dubious. To get a sense of the kind of flaw present in the argument, I would like to compare it with another argument. Suppose Matteo and Mattia both wear glasses. Now consider the argument:

Matteo can't see Mattia without using glasses.

therefore:

Matteo can't see Mattia unless Mattia is wearing glasses.

\footnotetext{
${ }^{1}$ http://www.mit.edu/ yablo/home/Papers_files/ex3.pdf
} 
This argument is clearly flawed: if Mattia takes off his glasses, Matteo can of course see him very well. The point is that glasses here play both the role of a necessary medium of Matteo's vision (Matteo sees through his glasses) and the role of possible object of Matteo's vision (Matteo can see Mattia with or without glasses). The important point is not to confuse the two roles: numbers, glasses, and perception could well be indispensable media of our vision or imagination, but this doesn't force us to consider them an indispensable part of the content of our vision and imaginations.

Let me finish by considering a funny example. One could argue that we cannot imagine ourselves dead because, in order to entertain such an imagination, we must be alive, after all. Sigmud Freud maid once a similar point. This seems to be another instance of the general pattern of reasoning I am considering:

P. Sigmud Freud cannot imagine himself without being alive.

therefore:

C. Sigmud Freud cannot imagine himself dead.

The argument for the existence of mathematical objects I considered at the beginning of this paper has a structure completely similar to the later ones. It should therefore be rejected for the same reasons. This means that reflection on the logic of imagination can help us to understand where popular arguments (both traditional and contemporary) go wrong. 